\section{Displacement sensor based on optical feedback interferometry in a GaN laser diode}

\author{
Jukka Hast, MEMBER SPIE \\ Leszek Krehut \\ Risto Myllylä, MEMBER SPIE \\ University of Oulu \\ Optoelectronics and Measurement Techniques \\ Laboratory and Infotech Oulu \\ Oulu 90014, Finland \\ E-mail: jukka.hast@ee.oulu.fi
}

\begin{abstract}
We describe an optical displacement sensor based on optical feedback interferometry in a blue-light emitting GaN laser diode. Also presented are preliminary results from measuring variations in the optical path length (OPL) of an external cavity (EC) in the 0 - to $240-\mathrm{nm}$ range. These results show that, within the specified range, the sensor follows linearly the OPL variation of the EC. Moreover, the slope between a reference and the measured OPL is 1.0003 , and the average deviation from the linear slope is $5 \mathrm{~nm}$ in this range. Finally, we also consider the stability of the interference signal in long-term measurements. (c) 2005 Society of Photo-Optical Instrumentation Engineers.
\end{abstract}

[DOI: 10.1117/1.2010167]

Subject terms: optical feedback; laser diode; optical path length; external cavity; blue wavelength.

Paper $050314 \mathrm{~L}$ received Apr. 21, 2005; accepted for publication Jun. 27, 2005; appeared online Jul. 1, 2005; published online Aug. 10, 2005.

\section{Introduction}

Optical feedback occurs when some part of emitted laser light is coupled back from an external reflector to a laser cavity, where it interacts with the original laser light producing an intensity modulation. This modulation can be detected by a photodetector placed on the opposite side of the laser cavity. A sensing scheme of this kind is simple, compact, and inexpensive, as there is only one optical axis to control and the only necessary component is the laser itself. As a result, optical feedback interferometry, also known as self-mixing or injection interferometry, has been used in a variety of vibration, displacement, and velocity measurement applications. ${ }^{1}$ In addition, it has been applied to several biomedical measurement applications such as cardiovascular diagnostics, measurements of arterial pulse wave shape and skin vibration and intra-arterial laser Doppler velocimetry. ${ }^{2-5}$

Typical light sources in optical feedback interferometry are single-mode laser diodes (LD) operating in the red or near-infrared wavelength range. ${ }^{1}$ This paper, however, uses a 405-nm GaN LD for optical feedback interferometry. The lower wavelength afforded by this diode improves the resolution of the optical feedback interferometer when detecting fringe shifts in displacement measurements. Earlier pa-

0091-3286/2005/\$22.00 @ 2005 SPIE pers on self-mixing interferometry have reported an accuracy of $40 \mathrm{~nm}$ in corresponding measurements. ${ }^{6,7}$

This paper describes an optical displacement sensor based on optical feedback interferometry in a GaN LD. Also presented are preliminary results from measuring variations in the optical path length (OPL) of an external cavity (EC) in the 0- to $240-\mathrm{nm}$ range. Finally, the paper also considers the stability of the interference signal in long-term measurements.

\section{Sensor Description}

The displacement sensor, constructed on a $12-\times 8-\mathrm{cm}$ aluminum board, consists of a LD and its driver, amplifiers, a temperature controller, and a digital computer interface. Its power supply is provided from an external source. As for the laser, it is a commercially manufactured GaN LD that emits blue light at $405 \mathrm{~nm}$ and has a threshold current of $47 \mathrm{~mA}$ at $21.0^{\circ} \mathrm{C}$. The emitted laser light is coupled back from an external reflector into the laser cavity, where it interacts with the original laser light, thereby producing an interference signal. This interference signal is then detected by a monitor photodiode located on the back side of the laser cavity. To amplify the interference signal, a dc-coupled preamplifier and an ac amplifier with a total transimpedance of $99 \mathrm{~dB} \Omega$ and a bandwidth of $13 \mathrm{~Hz}$ to $40 \mathrm{kHz}$ is used.

The temperature controller stabilizes the operating temperature of the $\mathrm{LD}$ with a resolution of $0.1^{\circ} \mathrm{C}$. Within the 1.5- to $10.6-\mathrm{mW}$ power range, the sensor's optical power stability is better than $0.2 \%$ and its average wavelength is $405.82 \pm 0.08 \mathrm{~nm}$. The LD driver, the temperature controller, and the sensor's data acquisition are all controlled by an ATMEGA128 onboard microprocessor, which is connected to a computer via an RS232 interface. In addition, the sensor also includes an analog interface for external data acquisition devices.

\section{Measurements and Results}

Preliminary measurements were performed to demonstrate the sensor's ability to measure variations in the OPL of the EC in the 0- to 240-nm range. To this end, collimated laser light was focused on a silicon mirror driven by a piezo transducer (PZT) with a displacement resolution of less than $\pm 5 \mathrm{~nm}$. The PZT, in turn, was driven with a ramp signal with a frequency of $10 \mathrm{~Hz}$ producing $650-\mathrm{nm}$ continuous modulation to the OPL of the EC. Then a dc offset was applied to the ramp signal, producing a change in the location where the new modulation signal of the EC starts.

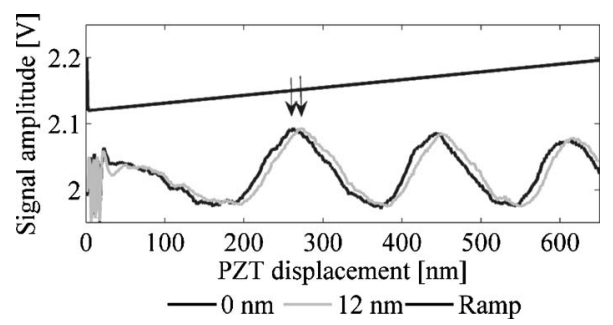

Fig. 1 Ramp signal and interference signals with a $0-\mathrm{nm}$ and 12-nm OPL offset of the EC. The arrows illustrate the 12-nm optical path difference between two signals. 
a)

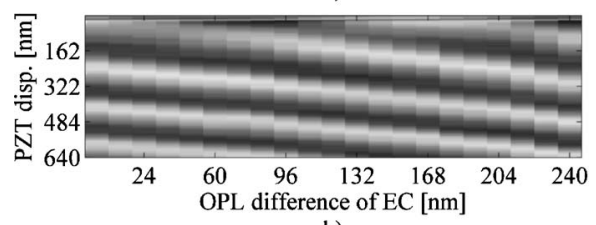

b)

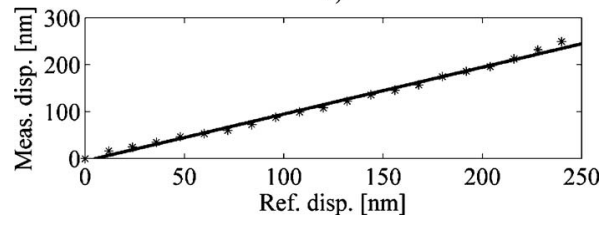

Fig. 2 (a) Fringe shifts as a function of the OPL of the EC. White color corresponds to increased intensity. (b) Linearity of the sensor.

Depending on the offset polarity, the OPL of the EC increases or decreases, producing a phase shift in the interference signal. Figure 1 presents the ramp signal and interference signals with a $0-\mathrm{nm}$ and $12-\mathrm{nm}$ OPL of the EC. As shown, a 12-nm offset shifts the interference signal to the right, whereas a negative offset shifts it to the left.

The OPL of the EC was changed from $0 \mathrm{~nm}$ to $240 \mathrm{~nm}$ with 12-nm steps. At each step, 20 measurements were conducted during a 2-s measurement time and the results were averaged. An intensity graph in Fig. 2(a) presents the fringe shifts of the interference signal as a function of the offset applied to the PZT. As seen, the fringe pattern of the interference signal shifts downwards, when the OPL of the EC changes. Each step is well detectable. In this range, the measured average shift was $12.5 \mathrm{~nm}$ and the mean standard deviation $3.7 \mathrm{~nm}$.

Figure 2(b) presents the linearity of the sensor. It shows the measured OPL of the EC as a function of the reference OPL applied to the EC via the PZT. To fit the linear slope to the data, a linear mean square approximation is used. The slope between the measured and reference values is 1.0003 , indicating that, within this range, linearity is very good. The average deviation of the measured values from the linear slope is $\pm 5.0 \mathrm{~nm}$, which is within the resolution of the PZT displacement.

The stability of the interference signal produced by the optical feedback interferometer is most strongly affected by temperature variations of the LD. Such variations have an effect on the length of the laser cavity and the lasing wavelength of the laser, thereby destabilizing the interference signal. In short-term measurements, lasting a few seconds, this temperature effect is negligible, but if a measurement takes several minutes or longer, the interference signal may start drifting. Figure 3 illustrates variations of the interference signal as a function of time. During the 3-min measurement depicted in this figure, the OPL of the EC was kept constant. The bold line presents the location of the maximum of the second fringe. As seen, the average location was at $302 \mathrm{~nm}$, but the line contains fluctuations with a standard deviation of $\pm 12.6 \mathrm{~nm}$ around the average. The maximum deviation from the average was $29.8 \mathrm{~nm}$.

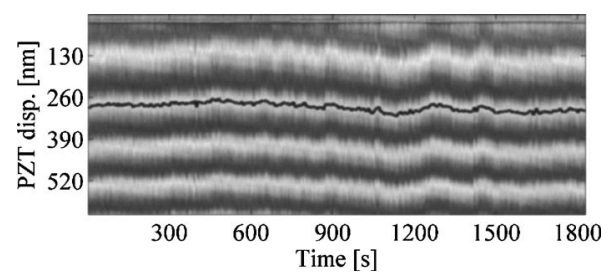

Fig. 3 Fluctuations in the interference signal due to temperature variations of the LD.

\section{Conclusion}

This paper presented an optical displacement sensor based on the optical feedback interferometry in a blue-light emitting GaN diode LD. First, the operation of the sensor was tested to detect the OPL variation of the EC in the 0 - to 240-nm range with $12-\mathrm{nm}$ steps. The measured average shift was $12.5 \mathrm{~nm}$, and the results show that the sensor operates linearly within the specified displacement range. Measured deviations from the linear slope were in the same class as the resolution of the PZT used to change the external optical path length. Next, the paper demonstrated the effect of temperature variations of the LD on the interference signal. It was found that the average shift of the interference signal was $\pm 12.6 \mathrm{~nm}$ in 3 -min measurements.

Optical feedback interferometry with blue lasers offers a potential readout technique to a variety of applications, particularly in the fields of biotechnology and nanotechnology. One example could be biosensors based on interferometry. Furthermore, in different MEMS devices, optical feedback interferometry may also prove very useful for purposes of controlling or collecting information concerning surrounding media. However, the use of GaN LDs is still limited by their relatively high price. In addition, GaN LD-based devices require high-gain electronics, as the sensitivity of the silicon monitor photodiodes is rather low at $405 \mathrm{~nm}$. Unfortunately, high-gain amplifiers may easily cause stability problems in the electronics and increase noise.

\section{References}

1. G. Giuliani, M. Norgia, S. Donati, and T. Bosch, "Laser diode selfmixing technique for sensing applications," J. Opt. A, Pure Appl. Opt. 4, 283-294 (2002).

2. K. Meigas, H. Hinrikus, R. Kattai, and J. Lass, "Self-mixing in a diode laser as a method for cardiovascular diagnostics," J. Biomed. Opt. 8(1), 152-160 (2003).

3. J. Hast, R. Myllylä, H. Sorvoja, and J. Miettinen, "Arterial pulse wave shape measurement using self-mixing effect in a diode laser," Quantum Electron. 32(11), 975-980 (2002).

4. A. Courteville, T. Gharbi, and J. Y. Cornu, "Noncontact MMG sensor based on the optical feedback effect in a laser diode," J. Biomed. Opt. 3(3), 281-285 (1998).

5. F. F. M. de Mul, L. Scalise, A. L. Petoukhova, M. van Herwijnen, P. Moes, and W. Steenbergen, "Glass-fiber self-mixing intra-arterial laser Doppler velocimetry: signal stability and feedback analysis," Appl. Opt. 41(4), 658-667 (2002).

6. S. Merlo and S. Donati, "Reconstruction of displacement waveforms with a single-channel laser diode feedback interferometer," IEEE J. Quantum Electron. 33, 526-531 (1997).

7. F. Gouraux, N. Servagent, and T. Bosch, "A phase-modulated method to improve the resolution of a self-mixing interferometer," Proc. IEEE-LEOS ODIMAP II, pp. 81-86 (1999). 